\section{Estado nutricional e consumo alimentar de adolescentes da rede pública de ensino da cidade de São Mateus do Sul, Paraná, Brasil}

\section{The nutritional status and dietary intake of adolescents in public schools in the city of São Mateus do Sul, in the State of Paraná, Brazil}

Renata Labronici Bertin 1

Elisa Noemberg Lazzari Karkle2

Anderson Zampier Ulbrich 3

Antônio Stabelini Neto4

Rodrigo Bozza5

Italo Quenni Araujo6

Wagner de Campos7
Abstract

Objectives: to characterize the nutritional status and dietary intake of adolescents attending public schools in the city of São Mateus do Sul, in the State of Paraná, Brazil.

Methods: the Body Mass Index was calculated and classified as proposed by the World Health Organization. Dietary intake was calculated using the Survey of Consumer Food Frequency. Analysis was carried out using descriptive and frequency analysis, and Student's t test with $p<0.05$.

Results: most individuals presented a normal nutritional status ( $84 \%$ being eutrophic, $12 \%$ overweight/obese and 4\% underweight). The diet was characterized by adequate energy intake, with a distribution of nutrients in accordance with recommendations and higher in boys than in girls. Ninety percent of the adolescents had an excessive intake of fatty acids. There was adequate intake of vitamin $C$ (287 $\mathrm{mg}$ and $328 \mathrm{mg}$ for boys and girls respectively) and iron (27 and $21 \mathrm{mg}$ respectively). The consumption of iron differed significantly between the genders $(t=3.765 ; p<0.001)$

Conclusions: the abnormalities found in dietary intake demonstrate the need for better understanding and evaluation of the type of food offered to students, as a way of developing strategies for intervention both within and outside of schools, and of helping prevent health problems related to nutrition.

Key words Adolescence, Nutritional status, Food consumption

\section{Resumo}

Objetivos: caracterizar o estado nutricional $e$ consumo alimentar de adolescentes da rede pública de ensino de São Mateus do Sul, Paraná, Brasil.

Métodos: o Índice de Massa Corporal foi calculado e classificado pela proposta da Organização Mundial da Saúde. O consumo alimentar foi obtido pelo Questionário de Freqüencia de Consumo Alimentar. Foi utilizada análise descritiva, de freqüencia e teste $t$ de Student com $p<0,05$.

Resultados: a maioria dos indivíduos apresentou estado nutricional normal (84\% eutróficos, $12 \%$ sobrepeso/obesidade e $4 \%$ baixo peso). A dieta caracterizou-se por consumo energético adequado, com distribuição de macronutrientes de acordo com as recomendações, sendo maior nos meninos do que nas meninas. Noventa por cento dos adolescentes consumiam ácidos graxos em excesso. Verificou-se consumo adequado de vitamina $C(287 \mathrm{mg}$ meninos e $328 \mathrm{mg}$ meninas) e ferro (27 e $21 \mathrm{mg}$, respectivamente). $O$ consumo de ferro diferiu estatisticamente entre os sexos $(t=3,765 ; p<0,001)$.

Conclusões: as anormalidades encontradas na alimentação evidenciam a necessidade de conhecer $e$ avaliar o tipo de alimentação oferecida aos alunos, a fim de elaborar estratégias de intervenção dentro e fora das escolas, além de auxiliar a prevenção de problemas de saúde relacionados à alimentação.

Palavras-chave Adolescência, Estado Nutricional, Consumo alimentar 


\section{Introdução}

A adolescência é uma etapa evolutiva que culmina todo o processo maturativo biopsicossocial do indivíduo. ${ }^{1}$ Cronologicamente, a adolescência corresponde ao período de 10 a 19 anos, sendo dividido em duas fases: fase 1-10 a 14 anos, e fase 2-15 a 19 anos. A faixa etária entre 10 a 14 anos inclui o início das mudanças puberais, e o término da fase de crescimento e desenvolvimento morfológicos ocorre no período de 15 a 19 anos. ${ }^{1}$ Esta fase caracteriza-se por um período de elevada demanda nutricional e por esse motivo, a nutrição desempenha papel fundamental no desenvolvimento do adolescente. ${ }^{2}$

As modificações que ocorrem na composição corporal dos adolescentes sofrem influências genéticas, ambientais, nutricionais, hormonais, sociais, culturais, e estão relacionadas com o aumento da massa corporal e o desenvolvimento físico, compreendendo também a maturação dos órgãos e sistemas para a aquisição de capacidades novas e específicas. 3

A nutrição está envolvida diretamente com essas modificações, principalmente com relação às recomendações nutricionais e aos padrões alimentares adotados por esta população. As necessidades energéticas aumentam com o rápido crescimento, com a maior proporção de massa corporal magra, com a menor proporção de gordura no organismo, com o aumento da atividade física, com o desenvolvimento muscular e com a maturação esquelética. ${ }^{4}$ Neste período da vida, vários fatores podem influenciar nas escolhas e hábitos alimentares, tais como valores socioculturais, imagem corporal, convivências sociais, situação financeira familiar, alimentos consumidos fora de casa, aumento do consumo de alimentos semipreparados, influência exercida pela mídia e disponibilidade de alimentos. ${ }^{3,4}$

Segundo Fisberg et al.,5 o comportamento alimentar do adolescente vincula-se fortemente aos padrões manifestados pelo grupo etário ao qual pertence, pela omissão de refeições, pelo consumo de alimentos de elevado conteúdo energético e pobre em nutrientes, pela ingestão precoce de bebidas alcoólicas e pelas tendências a restrições dietéticas. Para os autores, esses comportamentos fazem parte do estilo de vida dos adolescentes, e podem contribuir para as alterações no estado nutricional.

Torna-se necessário conhecer o comportamento alimentar dos adolescentes, devido ao fato de existir uma correlação positiva entre dieta e risco de morbimortalidade. Dietas inadequadas, com elevado teor de lipídios, energia e carboidratos simples, podem ser consideradas fatores de risco para doenças crônicas e obesidade. 6 Para Sichieri, ${ }^{7}$ essas mudanças no consumo alimentar, em conjunto com outras alterações no estilo de vida, especialmente aquelas relacionadas com a diminuição da prática de atividade física, podem estar contribuído para a elevação das taxas de prevalência de excesso de peso e obesidade nos diferentes grupos etários.

Vale destacar que o estado nutricional exerce influência nos riscos de morbimortalidade e no desenvolvimento e crescimento dos adolescentes, o que torna importante uma avaliação nutricional dessa população mediante procedimentos e diagnósticos que possibilitem identificar a magnitude, o comportamento e os determinantes dos agravos nutricionais, bem como identificar os grupos de risco.

A avaliação precisa do consumo alimentar e estado nutricional de adolescentes desperta grande preocupação, pois a formação de hábitos alimentares inadequados pode ocasionar desequilíbrios nutricionais desfavoráveis ao bom funcionamento do organismo. Esta pesquisa contribui com um diagnóstico inicial de uma realidade pouco explorada e aponta para a necessidade de estudos mais abrangentes que possam subsidiar a criação de políticas de atendimento para este grupo em estudo. Nesse contexto, o presente trabalho tem por objetivo caracterizar o estado nutricional e consumo alimentar de adolescentes de ambos os sexos, matriculados na rede pública de ensino, da cidade de São Mateus do Sul, Paraná.

\section{Métodos}

Trata-se de um estudo descritivo de corte transversal, com amostragem intencional, obtida a partir de uma população matriculada na rede pública de ensino da cidade de São Mateus do Sul, situada na região sul do Estado do Paraná, Brasil, com uma população segundo estimativa feita pela Fundação Instituto Brasileiro Geográfico de Estatística (IBGE), 8 o município possui 39.103 habitantes.

Para o cálculo do tamanho amostral, foram utilizados dados referentes ao número de alunos matriculados (1610 alunos sendo 787 meninos e 823 meninas) por sexo e faixa etária (12 a 16 anos) na rede pública de ensino na cidade de São Mateus do Sul, no ano de 2006, tais dados foram fornecidos pela Secretaria Municipal de Educação. De posse do número total de alunos matriculados, calculou-se a amostra de acordo com: a) número total de meninos e meninas; b) intervalo de confiança de $95 \%$; c) erro 
amostral de $5 \%$ e prevalência de $4,7 \%$, uma vez que a prevalência de excesso de peso encontrada em estudos com adolescentes brasileiros de ambos os sexos em diversas regiões do país variam de $4,7 \%$ a $22,9 \% .9,10$

A amostra intencional foi representada por dois colégios do município que têm maior representatividade da região urbana e rural, que correspondem a $1610(64,89 \%)$ do total de alunos matriculados na rede pública de ensino. Em cada escola os alunos foram selecionados de forma aleatória simples, sendo elegíveis todos com idades entre 12 a 16 anos. Os alunos selecionados foram indagados, através do preenchimento de um questionário, sobre o hábito de fumar, patologias existentes, utilização de medicamentos e sobre algumas questões relacionadas ao histórico médico de sua família. Caso o aluno sorteado não atendesse todos os critérios de inclusão para diminuir o número de variáveis intervenientes, como: a) nunca ter fumado; b) não apresentar histórico familiar de doenças cardíacas; c) não ser diabético; d) não apresentar algum outro tipo de patologia reconhecida que possa afetar os resultados do perfil lipídico; e) não ter ingerindo medicamento no dia da avaliação, o mesmo era descartado e em seu lugar um novo aluno era sorteado.

Desta forma, a amostra final foi composta por 170 adolescentes, sendo 63 do sexo masculino $(36,84 \%)$, e 107 do sexo feminino $(63,16 \%)$, representantes do oitavo e nono anos do ensino fundamental e primeiro ano do ensino médio, na faixa etária de 12 a 16 anos de idade, pertencentes aos estágios três e quatro de maturação sexual.

As avaliações foram realizadas no segundo semestre do ano de 2006, sendo que no seu início, foi realizado um estudo piloto antes do início da pesquisa, com 50 adolescentes de ambos os sexos, 25 meninos e 25 meninas, de uma escola da rede pública de ensino da cidade, a fim de testar os procedimentos metodológicos e os instrumentos da pesquisa.

Inicialmente, os adolescentes foram informados sobre o estudo, aqueles que concordaram em participar do estudo receberam um "termo de consentimento" para ser preenchido pelos pais ou respectivos responsáveis, no qual constava uma breve explicação dos objetivos da pesquisa, sobre os métodos que se-riam empregados e a afirmação que não haveria identificação dos mesmos.

Este estudo foi aprovado pelo Comitê de Ética em Pesquisa da Universidade Federal do Paraná, conforme a resolução 196/96 do Conselho Nacional de Saúde que envolve pesquisas em seres humanos, em conformidade com a declaração de Helsinque de 1975.
As avaliações foram realizadas na própria escola pré-selecionada, durante o período da manhã (das 8:00 às 12:00 horas). Todas as mensurações foram realizadas por uma equipe treinada composta por nutricionistas e professores de educação física do laboratório do Centro de Pesquisa de Exercício e Esporte (CPEE) do Departamento de Educação Física do Setor de Ciências Biológicas da Universidade Federal do Paraná (UFPR).

Para determinação do estágio de maturação sexual foi utilizado o método proposto por Tanner, 11 onde os estágios maturacionais se dividem de um a cinco, sendo o estágio 1 quando a criança se encontra no nível considerado pré-púbere e o outro extremo, o estágio 5 (pós-púbere), quando o processo maturacional está finalizado. O teste foi aplicado em forma de auto-avaliação do desenvolvimento da pilosidade pubiana.

Para avaliar o estado nutricional dos adolescentes, calculou-se o Índice de Massa Corporal (IMC), adotando como critério de classificação os valores para idade e sexo e os respectivos pontos de corte propostos pela World Health Organization (WHO). 12

Medidas de peso (em quilogramas) e altura (metros) foram obtidas em balança digital portátil da marca Plenna, com precisão de $100 \mathrm{~g}$, e em estadiômetro portátil da marca WCS, escalonado em $0,1 \mathrm{~cm}$.

Foram aferidas as dobras cutâneas tricipital e da panturrilha (ambas do lado direito) utilizando adipômetros da marca Cescorf $®$. As medidas foram realizadas em duplicata, considerando-se o valor médio para o cálculo. O percentual de gordura corporal foi calculado utilizando a fórmula de Slaughter et al., 13 considerando como excesso de gordura corporal os meninos que apresentaram percentual acima de $25 \%$ e as meninas, acima de $30 \%$.

As informações sobre o consumo alimentar foram obtidas usando-se o Questionário de Freqüência de Consumo Alimentar (QFCA), desenvolvido e validado para população brasileira por Sichieri e Everhart, 14 e posteriormente aplicado em outra pesquisa com adolescentes por Fonseca $e t$ al. 9

O questionário semiquantitativo utilizado para a coleta de dados sobre o consumo habitual de alimentos contém uma lista com 81 itens alimentares, no qual o entrevistado indicava o número de porções que costumava consumir e com que freqüência: mais de três vezes por dia; de duas a três vezes por dia; uma vez por dia; cinco a seis vezes por semana; duas a quatro vezes por semana; 
uma vez por semana; uma a três vezes por mês; nunca ou quase nunca.

A escolha desse instrumento para o estudo se deve pelo fato de alguns autores citarem o QFCA como um dos principais instrumentos metodológicos que relacionam a dieta à ocorrência de doença, por avaliar a ingestão alimentar de populações, ter boa reprodutibilidade e validade aceitável, além de ser mais prático, informativo, de fácil aplicação e baixo custo. 15

Os cálculos da ingestão calórica e composição da dieta dos participantes foram realizados através de uma planilha desenvolvida no Excel que, inicialmente, transforma as freqüências em freqüências diárias (utilizando-se a média de intervalo de freqüência); estas foram multiplicadas pelas quantidades das porções e, finalmente, as quantidades diárias foram transformadas em nutrientes. O cálculo dos macronutrientes e micronutrientes foi realizado através do banco de dados de composição de alimentos do Programa de Apoio à Nutrição, desenvolvido pela Escola Paulista de Medicina, Universidade Federal de São Paulo. 16

No tocante à análise qualitativa do Valor Energético Total (VET) da dieta, foram adotadas como referência as Dietary Reference Intakes (DRIs), recomendações preconizadas pelo Institute of Medicine (IOM), ${ }^{17}$ que considera aceitáveis, para indivíduos de 4 a 18 anos, os seguintes intervalos de participação de macronutrientes: $45-65 \%$ provenientes dos carboidratos, $25-35 \%$ dos lipídios, e de energia $2493,5 \mathrm{kcal}$. Para a análise do teor e qualidade das gorduras, adotaram-se as recomendações do Comitê de Nutrição da Academia Americana de Pediatria:18 ácidos graxos saturados $\leq 10 \%$ das gorduras totais, ácidos graxos poliinsaturados $\geq 10 \%$, e colesterol $\leq 300 \mathrm{mg} /$ dia. Para a análise qualitativa da prevalência de inadequação de ingestão de vitamina $\mathrm{C}$ e ferro optou-se por utilizar a Necessidade Média Estimada (Estimated Average Requirement, EAR) que estima a prevalência de inadequação de um nutriente por determinado grupo e é recomendada pelo Institute of Medicine. 19,20 Para a ingestão de cálcio, utilizou-se a Ingestão Adequada (Adequate Intake, AI), ${ }^{21}$ visto que não existe EAR estabelecido para esse nutriente.

Para manter melhor qualidade dos dados, foram excluídos os casos com consumo energético superior a $6000 \mathrm{kcal}$ ou inferior a $500 \mathrm{kcal}$.

$\mathrm{O}$ tratamento estatístico das informações foi realizado mediante o pacote computadorizado Statistical Package for the Social Science (SPSS), versão 11.0. Para caracterização da amostra utilizouse inicialmente a estatística descritiva (média e desvio padrão). Para determinar a diferença entre os sexos utilizou-se teste $t$ de Student, com nível de significância estipulado em $p<0,05$.

\section{Resultados}

Através dos critérios pré-estabelecidos para o desenvolvimento do estudo, do total elegível dos adolescentes avaliados, sete meninos e duas meninas foram excluídos por não terem contemplado adequadamente toda a bateria de testes proposta durante a pesquisa, e por apresentarem nos resultados do consumo alimentar valores subestimados e superestimados. Assim como, mais 15 indivíduos foram excluídos, sendo 4 por serem fumantes e 11 por apresentarem histórico positivo de fator de risco.

Desta forma a população estudada apresenta média de idade em anos de 15,0 $\pm 1,5$ e 14,8 $\pm 1,2$ para o sexo masculino e feminino, respectivamente. $\mathrm{O}$ valor médio do índice utilizado para classificação do estado nutricional foi de $20,4 \mathrm{~kg} / \mathrm{m}^{2} \pm 2,6$ e 20,1 $\mathrm{kg} / \mathrm{m}^{2} \pm 2,8$, de acordo com a mesma ordem de sexo. Com relação ao percentual de gordura, pôde-se observar diferença significativa entre os sexos $(t=-6,995, p=0,0001)$ (Tabela 1$)$.

Tabela 1

Descrição dos adolescentes em relação à idade e as medidas antropométricas peso, estatura, Indice de Massa Corporal (IMC) e percentual de gordura (\%G), estratificada pelo sexo.

\begin{tabular}{lcccc}
\hline Variáveis & \multicolumn{2}{c}{ Masculino } & \multicolumn{2}{c}{ Feminino } \\
\cline { 2 - 5 } & $\bar{X}$ & DP & $\bar{X}$ & DP \\
\hline Idade (anos) & 15,0 & 1,5 & 14,8 & 1,2 \\
Peso (kg) & 57,1 & 9,8 & 51,4 & 8,9 \\
Estatura (cm) & 166,7 & 8,1 & 159,5 & 6,9 \\
IMC (kg/m2) & 20,4 & 2,6 & 20,13 & 2,8 \\
$\%$ G & 16,48 & 7,1 & 23,47 & 5,7 \\
\hline
\end{tabular}

Quanto ao estado nutricional dos adolescentes do presente estudo verificou-se maior porcentagem, para ambos os sexos, de indivíduos dentro dos padrões de normalidade $(84,1 \%)$, enquanto que foi detectado que $12,3 \%$ dos participantes do estudo apresentaram sobrepeso/obesidade. As prevalências do estado nutricional de acordo com os percentis do IMC para ambos os sexos estão descrita na Tabela 2. 
Prevalência dos adolescentes de acordo com a classificação do estado nutricional segundo os percentis do Índice de Massa Corporal.

\begin{tabular}{|c|c|c|c|c|}
\hline \multirow[t]{2}{*}{ Estado nutricional } & \multicolumn{2}{|c|}{ Masculino } & \multicolumn{2}{|c|}{ Feminino } \\
\hline & $\mathrm{n}$ & $\%$ & $n$ & $\%$ \\
\hline Baixo Peso & 3 & 4,7 & 3 & 2,8 \\
\hline Eutrófico & 51 & 81,0 & 92 & 86,0 \\
\hline Sobrepeso & 3 & 9,5 & 11 & 10,3 \\
\hline Obesidade & 6 & 4,8 & 1 & 0,9 \\
\hline
\end{tabular}

Tabela 3

Descrição do consumo energético e de nutrientes da dieta, estratificado pelo sexo.

\begin{tabular}{|c|c|c|c|c|c|c|}
\hline \multirow[t]{2}{*}{ Nutrientes } & \multicolumn{2}{|c|}{ Masculino } & \multicolumn{2}{|c|}{ Feminino } & \multirow[b]{2}{*}{$t$} & \multirow[b]{2}{*}{$p$} \\
\hline & $\bar{x}$ & DP & $\bar{x}$ & DP & & \\
\hline Energia (kcal) & 2494,5 & 673,0 & 2299,4 & 571,8 & 2,015 & 0,046 * \\
\hline Carboidrato (g) & 344,0 & 97,1 & 325,7 & 79,7 & 1,336 & 0,183 \\
\hline Proteína (g) & 92,2 & 25,8 & 80,2 & 22,4 & 3,21 & $0,002 *$ \\
\hline Lipídio (g) & 83,2 & 29,6 & 75,0 & 26,1 & 1,873 & 0,063 \\
\hline Cálcio (mg) & 1200,7 & 427,9 & 1063,6 & 375,0 & 2,187 & $0,03 *$ \\
\hline Ferro (mg) & 27,1 & 10,5 & 21,3 & 9,1 & 3,823 & 0,001 * \\
\hline Vitamina C (mg) & 287,2 & 168,1 & 326,0 & 193,5 & $-1,326$ & 0,187 \\
\hline AGSA $(g)$ & 35,1 & 12,3 & 33,2 & 11,3 & 1,002 & 0,318 \\
\hline AGPO (g) & 11,2 & 5,1 & 11,2 & 5,2 & $-0,09$ & 0,928 \\
\hline Colesterol (mg) & 312,7 & 159,8 & 267,2 & 155,2 & 1,83 & 0,069 \\
\hline
\end{tabular}

*p<0,05; AGSA=Ácido Graxo Saturado; AGPO=Ácido Graxo Poliinsaturado.

Tabela 4

\begin{tabular}{|c|c|c|c|c|c|c|}
\hline \multirow[t]{2}{*}{ Nutrientes } & \multicolumn{3}{|c|}{ Masculino } & \multicolumn{3}{|c|}{ Feminino } \\
\hline & Insuficiente & Adequado & Elevado & Insuficiente & Adequado & Elevado \\
\hline Carboidrato (g) & 7,9 & 85,7 & 6,4 & 1,8 & 85,3 & 12,9 \\
\hline Proteína (g) & - & 100,0 & - & 0,9 & 99,1 & - \\
\hline Lipídio (g) & 7,9 & 73,0 & 19,1 & 6,5 & 49,1 & 44,4 \\
\hline Cálcio (mg) & 63,5 & 36,5 & - & 71,6 & 28,4 & - \\
\hline Ferro (mg) & - & 100,0 & - & 1,8 & 98,2 & - \\
\hline Vitamina C (mg) & 3,2 & 96,8 & - & 0,9 & 99,1 & - \\
\hline AGSA (g) & - & 11,1 & 88,9 & - & 9,3 & 90,7 \\
\hline AGPO (g) & 100,0 & - & - & 100,0 & - & - \\
\hline Colesterol (mg) & - & 61,9 & 38,1 & - & 70,6 & 29,4 \\
\hline
\end{tabular}

AGSA=Ácido Graxo Saturado; AGPO=Ácido Graxo Poliinsaturado. 
O consumo alimentar entre os adolescentes esta descrito na Tabela 3, porém, vale ressaltar que os valores elevados do desvio-padrão indicam que há grande variação de consumo entre os integrantes da amostra.

Quanto à diferença entre os sexos, foi observada diferença estatisticamente significativa para consumo de energia, proteína, cálcio e ferro.

Em relação à adequação dos nutrientes (Tabela 4), observou-se que para os macronutrientes o percentual de carboidratos estava de acordo com as recomendações em $85,7 \%$ dos meninos e $85,3 \%$ das meninas. Analisando-se o consumo de proteínas, observamos que $100 \%$ dos meninos e $99,1 \%$ das meninas estão com a ingestão adequada, alcançando a média recomendada para o grupo (10-30\% do valor energético total-VET), de acordo com o Institute of Medicine. 17 Quanto ao consumo de lipídeos, observa-se que $73 \%$ dos meninos e $49,1 \%$ das meninas estão com ingestão adequada, e $19 \%$ e $44,4 \%$ respectivamente apresentaram consumo superior ao recomendado para o grupo ( $>35 \%$ do valor energético total).

$\mathrm{Na}$ análise referente à qualidade dos lipídeos da dieta alimentar, identificamos que a maior proporção de lipídeos ingeridos é proveniente dos ácidos graxos saturados, sendo que $88,9 \%$ dos adolescentes e $90,7 \%$ das adolescentes apresentam consumo superior ao recomendado. 20

\section{Discussão}

Ao se verificar na presente pesquisa que a maior parte dos avaliados estavam dentro de um estado nutricional adequado aos padrões de normalidade $(84,1 \%)$ e que $12,3 \%$ dos indivíduos acima do peso, algumas pesquisas realizadas no Brasil mostraram a proporção de sobrepeso/obesidade superior ao encontrado nesse estudo. $8,22,23$ No entanto, nem todos os estudos utilizaram os mesmos critérios de classificação do estado nutricional; tal apontamento merece destaque pela necessidade de se adotar uma padronização de classificação no referido grupo, obtendo-se assim uma melhor fidedignidade na comparação entre os estudos.

Quanto à proporção de indivíduos com sobrepeso encontrada em nosso estudo, embora esta seja inferior à da literatura, ela pode ser representativa para cidades com este número de habitantes, pois diante da transição nutricional que o Brasil vem passando nas últimas duas décadas, como o declínio da desnutrição e aumento do excesso de peso entre os adolescentes, esta prevalência observada nesta cidade já pode ser considerada preocupante.

Segundo dados da Pesquisa sobre Padrões de Vida (PPV), realizada em 1996/1997, a prevalência de sobrepeso e obesidade atualmente é de $14 \%$ em crianças e adolescentes na faixa etária de 6 a 18 anos. ${ }^{24}$ Estudos realizados em algumas cidades brasileiras mostram que o sobrepeso e a obesidade já atingem mais de $20 \%$ das crianças e adolescentes, como em Recife, alcançando $35 \%$ dos escolares avaliados. 25 Desta forma, enfatiza-se a necessidade de intervenção precoce no estágio de sobrepeso, tendo em vista que adolescentes com sobrepeso e/ou obesidade estão mais propensos a desenvolverem problemas de saúde como diabetes, hipertensão e doenças cardiovasculares, além de terem maiores chances de se tornarem adultos obesos. 26

A diferença significativa da gordura corporal entre meninos e meninas é esperada pela idade dos adolescentes. Crianças pré-puberes partem de um percentual de gordura de aproximadamente $15 \%$ (meninos) e 19\% (meninas) e até os 20 anos atingem percentual de gordura de $12 \%$ (meninos) e $23 \%$ (meninas). 27 A média de idade das meninas avaliadas $(14,8)$ é superior à idade média da menarca no Brasil (cerca de 12 anos), assim as meninas já teriam passado por alterações importantes em sua composição corporal.27

Quanto ao valor energético e à distribuição dos macronutrientes das dietas, observa-se uma tendência em ambos os grupos de uma ingestão adequada. Os valores médios encontrados para o consumo energético nesse estudo são semelhantes aos encontrados em outros estudos. 14,25

Segundo o Institute of Medicine, 17 a avaliação do consumo energético deve ser feita com base neste Instituto. Como o IMC médio da população está dentro da faixa de percentis desejável, conclui-se que a média de ingestão calórica está adequada para a manutenção de um peso saudável.

Desta forma, dados semelhantes também foram encontrados em pesquisa realizada por Zalilah et al..28 que ao avaliarem 618 adolescentes da Malásia (317 do sexo feminino e 301 do sexo masculino), verificaram consumo médio de energia de $1903 \mathrm{kcal}$ para as meninas eutróficas e $2133 \mathrm{kcal}$ para os meninos eutróficos, sendo que a distribuição de macronutrientes do consumo energético foi de 32$34 \%, 51-53 \%, 14-16 \%$ de gordura, carboidrato e proteínas respectivamente.

Em relação ao consumo protéico, os adolescentes não apresentaram alto consumo deste nutriente. Resultados semelhantes foram encontrados em estudo realizado por Caroba e Silva,29 ao avaliarem o consumo alimentar de 578 adolescentes 
matriculados em escolas públicas de Piracicaba, São Paulo, em que o consumo médio de proteínas estava na faixa recomendada. Por outro lado, em estudo realizado por Kazapi et. al., 30 envolvendo 797 estudantes de escolas públicas e privadas da cidade de Florianópolis, Santa Catarina, verificaram que 50\% dos adolescentes de ambas as redes de ensino apresentaram um alto consumo de proteínas. Albano e Souza $^{23}$ observaram que os valores médios encontrados atenderam $223,96 \%$ da recomendação para o sexo masculino e $176,99 \%$ para o sexo feminino, indicando também um alto consumo desse nutriente.

Vale destacar que, mesmo alguns estudos mostrando a ingestão de proteínas por adolescentes acima dos valores recomendados, não permitem concluir com exatidão se o consumo está sendo excessivo para o adolescente. Segundo Vitolo, ${ }^{31}$ para concluir se o perfil protéico da dieta é suficiente ou não, se não está excessivo, ou se o adolescente deve alterar a qualidade da proteína da dieta, faz-se necessário considerar alguns parâmetros como sexo, composição corporal, atividade física e estágio pubertário.

Com relação ao consumo de lipídeos, mesmo não tendo observado uma alta prevalência de consumo no grupo em geral, ao avaliarmos a qualidade da gordura, observou-se que a maior parte da gordura consumida na dieta é proveniente dos ácidos graxos saturados. Dados semelhantes foram encontrados em estudo realizado por Lima et al., 32 em que os autores verificaram ingestão de lipídios no limite máximo aceitável e um percentual de consumo de ácidos graxos saturados próximo ao dobro do recomendado.

Tais resultados merecem atenção, visto que o consumo excessivo de lipídios na forma de gorduras saturadas é o principal fator determinante na elevação das concentrações plasmáticas de LDL colesterol. As gorduras saturadas inibem a depuração plasmática de LDL-c, além de permitir maior entrada de colesterol nessas partículas e diminuir sua remoção do plasma. ${ }^{33}$

No tocante ao consumo de ferro, não foi observado, no grupo estudado, consumo abaixo do recomendado, mas a média encontrada foi superior aos encontrados em outras pesquisas. Caroba e Silva $^{29}$ verificaram média de ingestão de $13,9 \mathrm{mg}$. Albano e Souza 23 defrontaram-se com consumo médio de 13,3 mg para o sexo masculino e 12,13 mg para o sexo feminino. Sichieri7 constatou consumo mediano de $17,7 \mathrm{mg}$ para meninos e $16,1 \mathrm{mg}$ para meninas.

O consumo adequado de ferro na adolescência é fundamental, pois esse mineral participa do trans- porte de oxigênio, produção de energia por meio da síntese de ácidos nucléicos, co-fator em reações enzimáticas e vários outros processos metabólicos.26,31,34 $\mathrm{Na}$ adolescência, em decorrência do rápido crescimento, do aumento da massa muscular, do volume sanguíneo e das enzimas respiratórias, as necessidades desse mineral são maiores para esse grupo.

O inadequado consumo de cálcio encontrado nesse estudo vai ao encontro com dados encontrados em outras pesquisas.22,23 Em contrapartida, no estudo realizado por Greene-Finestone et al.,35 a média de consumo de cálcio nas dietas com porcentagem de carboidratos entre $45-65 \%$, foram maiores do que as encontradas no presente estudo.

Segundo a WHO,26 o consumo deficiente de cálcio é encontrado em diversos países desenvolvidos e a prevalência da deficiência é duas vezes maior em meninas que em meninos. Os dados levantados acompanham essa tendência, apontando menor média de ingestão entre as adolescentes do sexo feminino. O menor aporte de alimentos (pela menor necessidade energética, comparadas aos meninos) acaba limitando, nesse grupo, a ingestão de micronutrientes como um todo e implica na necessidade de uma escolha mais criteriosa.

Nesse contexto, os dados apontados na literatura são preocupantes, pois no Brasil há uma aparente tendência de redução no consumo de leite, o qual vem sendo substituído por refrigerantes e outras bebidas industrializadas. Essa tendência pode repercutir negativamente sobre o desenvolvimento ósseo dos adolescentes, pois o baixo consumo de cálcio pode ser um fator limitante para o crescimento linear e mineralização óssea, favorecendo o aparecimento da osteoporose. 26,31

Avaliando o consumo de vitamina $\mathrm{C}$, todos atingiram o consumo preconizado pelo IOM. 18 Resultados semelhantes foram encontrados por Greene-Finestone et al., 35 que constataram consumo médio de 243, $5 \mathrm{mg}$ para os meninos e 214,7 mg para as meninas. Albano e Souza ${ }^{23}$ verificaram consumo de $120 \mathrm{mg}$ para o sexo masculino e $161 \mathrm{mg}$ para o sexo feminino.

Sabe-se que, apesar da deficiência na ingestão de vitamina $C$ não ser considerada problema entre os adolescentes, essa vitamina tem sua importância, pois atua como agente redutor em várias e importantes reações de hidroxilação no organismo, participa na cicatrização, na formação dos dentes e na integridade dos capilares. 31 Nesse contexto, devemos destacar que a ingestão insuficiente de vitamina $\mathrm{C}$ é preocupante somente para os adolescentes que evitam o consumo de alimentos fontes, além 
daqueles que fumam e/ou usam contraceptivos orais. 31

Cabe destacar que várias metodologias vêm sendo utilizadas para avaliar o consumo alimentar de indivíduos jovens, no sentido de obter dados válidos, reprodutíveis e comparáveis. Porém como a amostra foi convidada aleatoriamente a participar do estudo dentro dos requisitos abordados, algumas outras limitações podem estar contidas nesta seleção, assim como, as que foram observadas durante a avaliação, como a habilidade do indivíduo em fornecer informações exatas e precisas quando questionadas e a própria limitação do tipo de inquérito dietético utilizado.

A população estudada encontra-se majoritariamente com o estado nutricional dentro dos padrões de normalidade. A dieta dos adolescentes é caracterizada por aporte energético adequado, com distribuição de macronutrientes de acordo com as recomen-

\section{Referências}

1. OMS (Organizacion Mundial de la Salud). La salud de los jóvenes: un reto y una esperanza. Geneva; 1995.

2. Albano RD, Souza SB. Ingestão de energia e nutrientes por adolescentes de uma escola pública. J Pediatr. (Rio J). 2001; 77: 512-6.

3. Bianculli $\mathrm{CH}$. Crecimiento físico y endocrinología em la pubertad. In: OPS (Organización Panamericana de la Salud). La salud del adolescente y del joven. Washington, DC; 1995. p. 87-94.

4. Carruth BR. Adolescência. In: OPS (Organización Panamericana de la Salud). Conocimientos actuales sobre nutrición. 6. ed. Washington, DC; 1991. p. 375-84.

5. Fisberg M, Bandeira CRS, Bonilla EA, Halpern G, Hirschbruch MD. Hábitos alimentares na adolescência. Pediatr Mod. 2000; 36: 724-34.

6. Andersen LF, Nes M, Sandstad B, Bjorneboe GE, Drevon CA. Dietary intake among Norwegian adolescents. Eur J Clin Nutr. 1995; 49: 555-64.

7. Sichieri R. Avaliação do consumo alimentar e do consumo de energia. In: Sichieri R, organizador. Epidemiologia da obesidade. Rio de Janeiro: Ed. EDUEJ; 1998. p. 65-88.

8. (IBGE) Fundação Instituto Brasileiro de Geografia e Estatística. Censo demográfico 2002: estimativa para população brasileira para o ano de 2006 [monografia online] [citado em: 26 jan. 2006].. Disponível em: http://ibge.gov.br

9. Fonseca VM, Sichieri R, Veiga GV. Fatores associados à obesidade em adolescentes. Rev Saúde Pública. 1998; 32 : 541-9.

10. Guedes DP, Guedes JERP, Barbosa DS, Oliveira JA, Stanganelli LCR. Fatores de risco cardiovasculares em adolescentes: indicadores biológicos e comportamentais. Arq Bras Cardiol. 2006; 86: 439-50. dações. O consumo de vitamina $\mathrm{C}$ e ferro está adequado, porém há consumo insuficiente de cálcio. Há também excesso de ingestão de ácidos graxos saturados.

Tal realidade evidencia a necessidade de novos estudos para o monitoramento do consumo alimentar de adolescentes, tendo em vista a importância da implantação de ações de educação nutricional focando a qualidade da dieta, promovendo assim uma atitude de prevenção a ser assumida por toda a vida.

Vale ressaltar que, diante do papel fundamental que a escola ocupa na tarefa de educar e informar sobre hábitos alimentares saudáveis, é importante conhecer, comparar e avaliar o tipo de alimentação que é oferecido aos alunos, pois tal conhecimento pode ser subsídio para a elaboração de estratégias de intervenção, dentro e fora das escolas, além de poder auxiliar na prevenção de problemas de saúde, relacionados com a alimentação.

11. Tanner JM. Growth and adolescence. Oxford: Blackwell Scientific; 1962.

12. WHO (World Health Organization). Physical status: the use and interpretation of antropometry. Geneva; 1995.

13. Slaughter MH, Lohman TG, Boileau RA, Horswill CA, Stillman RJ, Van Loan MD, Bemben, DA. Skinfold equations for estimation of body fatness in children and youth. Hum Biol. 1988; 60: 709-23.

14. Sichieri R, Everhart JE. Validity of a Brazilian food frequency questionnaire against dietary recalls and estimated energy intake. Nutr Res. 1998; 18: 1649-59.

15. Salvo VLMA, Gimeno SGA. Reprodutibilidade e validade do questionário de freqüência de consumo alimentar. Rev Saúde Pública. 2002; 36: 505-12.

16. Universidade Federal de São Paulo. Escola Paulista de Medicina. Programa de apoio à nutrição. São Paulo; 1995.

17. IOM (Institute of Medicine). Dietary reference intakes for energy, carbohydrate, fiber, fatty acids, cholesterol, protein and amino acids Food and Nutrition Board. Washington, DC: National Academy Press; 2002.

18. American Academy of Pediatrics Committee of Nutrition. Statement on cholesterol. Pediatrics. 1992; 90: 469-73.

19. IOM (Institute of Medicine). Dietary reference intakes for vitamin C, vitamin E, selenium, and carotenoids. Washington, DC: National Academy Press; 2000.

20. IOM (Institute of Medicine). Dietary reference intakes for vitamin A, vitamin K, arsenic, boron, chromium, copper, iodine, iron, manganese, molybdenum, nickel, silicon, vanadium and zinc. Washington, DC: National Academy Press; 2001.

21. IOM (Institute of Medicine). Dietary Reference Intakes for Calcium, Phosphorus, Magnesium, Vitamin D, and Fluoride. Washington, DC: National Academy Press; 1997. 
22. Garcia GCB, Gambardella AMD, Frutuoso MFP. Estado nutricional e consumo alimentar de adolescentes de um centro de juventude da cidade de São Paulo. Rev Nutr. 2003; 16: 41-50

23. Albano RD, Souza SB. Estado nutricional de adolescentes: "risco de sobrepeso" e "sobrepeso" em uma escola pública do município de São Paulo. Cad Saúde Pública. 2001; 17 : 941-7.

24. Wang Y, Monteiro C, Popkin BM. Trends of obesity and underweight in older children and adolescents in the United States, Brazil, China and Russia. Am J Clin Nutr. 2002; 75 : 971-7.

25. Balaban G, Silva GAP. Prevalência de sobrepeso em crianças e adolescentes de uma escola da rede privada de Recife. J Pediatr (Rio J). 2001; 77: 96-100.

26. WHO (World Health Organization). Nutrition in adolescence: issues and challenges for the health sector: issues in adolescence health and development. Geneva; 2005.

27. Vitalle MSS, Yomioka CY, Juliano Y, Amâncio OMS Índice de Massa Corporal, desenvolvimento puberal e sua relação com a menarca. Rev Assoc Med Bras. 2003; 49 429-33.

28. Zalilah MS, Khor GL, Mirnalini K, Norimah AK, Ang M. Dietary intake, physical activity and energy expenditure of Malaysian adolescents. Singap Med J. 2006; 47: 491-8.
29. Caroba DCR, Silva MV. Consumo alimentar de adolescentes matriculados na rede pública de ensino de Piracicaba-SP. Segurança alimentar e nutricional. Rev Nutr PUCCAMP. 2005; 12: 55-66.

30. Kazapi IM, Di Pietro PF, Avancini SRP, Freitas SFT, Tramonte VLCG. Consumo de energia e macronutrientes por adolescentes de escolas públicas e privadas. Rev Nutr. 2001; 14 (Supl): 27-33.

31. Vitolo MR. Recomendações dietéticas para adolescentes. In: Vitolo MR, organizador. Nutrição: da gestação à adolescência. Rio de Janeiro: Reichmann; 2003. p. 206-15.

32. Lima SCVC, Arrais RF, Pedrosa LFC. Avaliação da dieta habitual de crianças e adolescentes com sobrepeso e obesidade. Rev Nutr PUCCAMP. 2004; 17: 469-77.

33. Rique ABR, Soares EA, Meirelles CM. Nutrição e exercício na prevenção e controle das doenças cardiovasculares. Rev Bras Med Esporte. 2002; 8: 1-11.

34. Assao TY, Silva DG, Ribeiro LC, Devincenzi MU, Sigulem DM. A importância do ferro na saúde e nutrição do grupo materno infantil. Compacta Nutr. 2004; 5: 7-22.

35. Greene-Finestone LS, Campbell MK, Evers SE, Gutmanis IA. Adolescent's low-carbohydrate-density diets are related to poorer dietary intakes. J Am Diet Assoc. 2005; 105: 1783-6.

Recebido em 9 de maio de 2007

Versão final apresentada em 4 de setembro de 2008

Aprovado em 10 de setembro de 2008 